\title{
Role of Peripheral Nerve Stimulation in Degenerative Lumbar Spine Pathologies with Early Neurological Deficit
}

\author{
Saurabh R Muni ${ }^{1}$, Sunil Bhosle ${ }^{2}$, Kinjal R Muni ${ }^{3}$, Siddarth Virani $^{4}$
}

\begin{abstract}
Degeneration is derived from the Latin word "degenerare" meaning to depart from one's own kind. Degeneration literally means "deterioration of a tissue or an organ in which its function is diminished or its structure is impaired Patients with degenrative back pain are common in orthopaedic opd, of these patients with early neurological deficit (MRC Grade>2) with only PID or LCS excluding those with instability were divided into two groups, 1 st receiving bed rest, traction and medications and 2 nd receiving nerve stimulation using faradic current in addition to above modalities. Most of the Patients of group 2 didn't only recover but also recovery was hastened by the use of PNS. Thus concluding that PNS is an effective additional conservative treatment modality in treating such pathologies and avoiding unnecessary surgeries in many of them.
\end{abstract}

Keywords: Early neurological deficit, PID/LCS , Peripheral nerve stimulation, Faradic current, Hastening and increasing recovery rate

\section{Introduction}

Degenerative disorders are most common in the lumbar and cervical spine ${ }^{[1] .}$ Degeneration is derived from the Latin word "degenerare" meaning to depart from one's own kind. Degeneration literally means "deterioration of a tissue or an organ in which its function is diminished or its structure is impaired". Clinical examination is an important aspect in diagnosing degenerative spine disorders. A complete neurological examination is necessary to pick up subtle signs like restricted SLR, power loss in single muscle etc.

Most of these clinical conditions associated with neurodeficit are lumbar canal stenosis; prolapsed intervertebral disc, which are usually treated in conservative manner with lumbar traction which helps to immobilize the patient in bed more than its literal meaning of giving traction. In many of these steroids or other antiinflammatory agents are administered to alleviate the local root compression due to edema \& swelling which is the cause of neurodeficit ${ }^{[1,2,3]}$.

But while the patient recovers from the pathology the involved motor units of the muscle undergo atrophy \& wasting, which when the nerve recovers gives the poor functional outcome to the patient. So hereby the application of peripheral nerve stimulation will help in maintaining the properties of Neuro-Muscuar junction \& helps to give the patient a better functional outcome ${ }^{[4,5]}$.

Peripheral nerve stimulation is the method in which specific motor points of the muscle are stimulated using a stimulator which gives a Faradic current the intensity of which can be modulated. This method of PNS will help in extending the conservative management $\&$ help in better recovery of the patient

In peripheral nerve disorders like bells palsy ${ }^{[3]}$ its role is already well established. In this study we would like to explore the same usefulness of nerve stimulation in spinal pathologies ${ }^{[6]}$ with neurodeficit like Prolapsed Intervertebral Disc or Lumbar Canal Stenosis.

\section{Literature Survey}

In the general population, the incidence of low back pain and early neurological deficit due to degenerative changes and neck pain is equally distributed between men and women. But in the working population the incidence is more in men as men are more involved in heavy work.

An individual muscle is made up of many motor units of different types, giving the muscle its particular characteristics. Motor unit is consists of an anterior horn cell, alpha motor neuron and all muscle fibers it supplies.

The number of muscle fibers supplied by motor neuron varies according to the function of the muscle (fine movements like eye movement needs few numbers while postural muscles need large number). During voluntary contraction of muscle there is smooth contraction due to asynchronous firing of motor neuron.

Force of contraction is graded by an increase in the number of motor unit recruited (spatial summation) and increase in frequency of nerve impulse (temporal summation). Strength muscles as shown by Hon Sun Lai et al. (1988) that electrical stimulation of muscles for 3 weeks gain more muscle strength in group treated with high intensity current than other group treated with low intensity current. The force of isometric contraction showed greater gain than that of concentric contraction. Eccentric contraction showed no significant gain.

Mechanism by which the peripheral nerve stimulation acts.

1) Reduction of muscle atrophy by maintenance of protein synthesis in muscle rather than preventing protein breakdown in cases of inhibited muscle contraction due to pain (as to stimulate quadriceps muscle after knee injury or surgery) or during splinting to maintain muscle power.

2) Electrical stimulation of innervated muscle will lead to increase metabolism and blood flow (increase oxygen uptake, carbon dioxide, lactic acid, and local temperature). 


\section{International Journal of Science and Research (IJSR) \\ ISSN (Online): 2319-7064 \\ Index Copernicus Value (2013): 6.14 | Impact Factor (2015): 6.391}

3) Increasing venous return \& lymphatic flow thereby reducing the edema.

The current used for stimulation of innervated muscle is faradic current or similar electrical pulses applied to cause muscle contraction Eutrophic electrotherapy is the record of frequencies of the motor unit action potential by electromyogram and used to determine the frequency of the muscle to improve the functional ability. Eutrophic electrotherapy used in Bell's palsy and applied to quadriceps muscles of elderly subjects with disuse atrophy.

In 1987, Farragher et al ${ }^{[7]}$ approached the issue of chronic paralysis of the facial nerve from a different perspective. These investigators focused on stimulating surviving motor units as well as sensory nerve fibers rather than denervated fibers of the facial muscles. To achieve adequate excitation of intact motor units, they used stimulus parameters based on the normal firing characteristics of the facial nerve

Four trials studied the efficacy of electrical stimulation (313 participants), three trials studied exercises (199 participants), and five studies combined some form of physical therapy and compared with acupuncture (360 participants). There is evidence from a single study of moderate quality that electrical stimulation is beneficial to people with chronic facial palsy when compared with controls and from another low quality study that it is possible that facial exercises could help to reduce synkinesis (a complication of Bell's palsy), and the time to recover.

Study by Scott et al. (1988) showed that similar low frequency stimulation of lateral popliteal nerve with sufficient intensity to help with cases of foot drop. Studies by Rutherford et al and Cramp et al shows efficacy of nerve stimulation in cases of loss of muscle range due to trauma and splinting.

\section{Methodology}

This was a prospective study (pilot study) with sample size of 50 divided into two groups.

\section{Inclusion Criteria}

Patients aged above 20 with radiculopathy secondary to degenerative lumbar spine disorder. Those with Early Neurodeficit which is defined as isolated nerve root involvement with power graded by MRC (Medical research council) grading as 3 or 4 .

\section{Exclusion Criteria}

Patients with dense neurodeficit, those with medical conditions confounding the diagnoses e.g Diabetes Mellitus or those that may interfere with treatment e.g. metallic implants. The patients were divided into two groups

Group A (Control): Which received standard modality of treatment with lumbar traction along with a course of intravenous steroids.

Group B (Test): Which in addition received peripheral nerve stimulation along with standard modality of treatment with lumbar traction along with a course of intravenous steroids. Each patient was to be stimulated using peripheral nerve stimulator everyday for 3 weeks (maximum interval).

\section{Methodology of Stimulation}

To apply stimulation over the desired muscle, electrode was placed over the motor point of the muscle $\&$ the operators hand was in contact with the patients tissues so that the contractions could be felt. A fixed surge \& duration were selected \& kept constant \& stimulation was started with minimal intensity which was gradually increased until a good muscle contraction was obtained at the maximum point of each surge $\&$ the surging is continued to produce alternate contraction \& relaxation of muscle. When the current is surged the contraction gradually increases and decreases in strength, in a manner similar to voluntary contraction.

Patients were stimulated while being admitted in ward \& at each visit the intensity of current required to initiate a contraction was noted. Decrease in minimal current intensity which initiated a contraction was taken as improvement. Duration of stimulation was maximum upto 3 weeks.

Stimulation was stopped prior to 3 weeks in case. Patient improved neurologically to grade 5, or worsened neurologically or wanted to opt out.

\section{Results \& Discussions}

Neurological status of patients in control group

\begin{tabular}{|c|c|c|c|}
\hline $\begin{array}{c}\text { Neurostatus } \\
\text { Motor Grade } \\
\text { (Post) }\end{array}$ & \multicolumn{2}{|c|}{$\begin{array}{c}\text { Neurostatus Motor Grade } \\
\text { (Pre) }\end{array}$} & \multirow{2}{*}{ Total } \\
\cline { 2 - 4 } 3 or 3+ & $3 / 3+$ & 4 & \\
\hline \multirow{2}{*}{4 or $4+$} & 4 & 0 & 4 \\
\hline \multirow{2}{*}{5} & $43.33 \%$ & $0.00 \%$ & $16.00 \%$ \\
\hline \multirow{2}{*}{ Total } & 5 & 8 & 13 \\
\cline { 2 - 4 } & $25.67 \%$ & $61.54 \%$ & $52.00 \%$ \\
\hline & 12 & 5 & 8 \\
\hline
\end{tabular}

p- value -0.07

Neurological status of patients in test group

\begin{tabular}{|c|c|c|c|}
\hline \multirow{2}{*}{$\begin{array}{c}\text { Neurostatus Motor } \\
\text { Grade (Post) }\end{array}$} & \multicolumn{2}{|c|}{ Neurostatus Motor Grade (Pre) } & \multirow{2}{*}{ Total } \\
\cline { 2 - 3 } 3 3 or 3+ & $3 / 3+$ & 4 & \\
\cline { 2 - 4 } & $33.33 \%$ & $0.00 \%$ & $0.00 \%$ \\
\hline \multirow{2}{*}{4 or $4+$} & 1 & 5 & \\
\cline { 2 - 4 } & $8.33 \%$ & $38.46 \%$ & $0.00 \%$ \\
\hline \multirow{2}{*}{5} & 7 & 8 & \\
\cline { 2 - 4 } & $58.33 \%$ & $61.54 \%$ & $0.00 \%$ \\
\hline \multirow{2}{*}{ Total } & 12 & 13 & 25 \\
\cline { 2 - 4 } & $100.00 \%$ & $100.00 \%$ & $100.00 \%$ \\
\hline
\end{tabular}

\section{p- value $\mathbf{- 0 . 0 3}$}

Comparison of the two tables shows that there is significant differnce in post treatment neurological status of patients with $p$ value of 0.03 after comparing both the groups showing that the use of peripheral nerve stimulation produces statistically significant improvement in patients with test group as compared to controls, proving its efficacy 


\section{International Journal of Science and Research (IJSR) \\ ISSN (Online): 2319-7064}

Index Copernicus Value (2013): 6.14 | Impact Factor (2015): 6.391

as a good additional conservative modality of treatment for degenerative lumbar spine pathologies.

The reason for improvement in test group is due to the biologic effects that the nerve stimulation generates in the form of conduction across the neuro -muscular junction and axoplasmic flow across the nerve membrane.

Many other studies have demonstrated an increased blood flow, for example Currier at al. ${ }^{[8]}$ (1986). Using 10 to $30 \%$ of maximum voluntary contraction these author quantified a $20 \%$

Mean duration of improvement in both the groups.

\begin{tabular}{|c|c|c|c|c|}
\hline Mean & Group & Mean & SD & p-value \\
\cline { 2 - 4 } Duration for & Control & 15.52 & 4.4 & $<0.01$ \\
\cline { 2 - 4 } Improvement & Test & 8.28 & 4.31 & \\
\hline
\end{tabular}

Mean duration of improvement in control was approximately 15 days while that in test group was 8 days, which is statistically significant with a $\mathrm{p}$ value of $<0.01$. This manifests that not only PNS helps in recovery as already discussed above it also helps in hastening the recovery as compared with the control groups.

\begin{tabular}{|c|c|c|c|}
\hline \multirow{2}{*}{ Improvement } & \multicolumn{2}{|c|}{ Group } & \multirow{2}{*}{ Total } \\
\cline { 2 - 4 } & Control & Test & \\
\hline \multirow{2}{*}{ No } & 15 & 8 & 21 \\
\cline { 2 - 4 } & $60.00 \%$ & $32.00 \%$ & $42.00 \%$ \\
\hline \multirow{2}{*}{ Yes } & 10 & 17 & 23 \\
\cline { 2 - 4 } & $40.00 \%$ & $68.00 \%$ & $46.00 \%$ \\
\hline \multirow{2}{*}{ Total } & 25 & 19 & 44 \\
\cline { 2 - 4 } & $100.00 \%$ & $76.00 \%$ & $88.00 \%$ \\
\hline \multicolumn{3}{|c}{$\mathrm{p}$-value - 0.047} & \\
\hline
\end{tabular}

\section{Conclusions}

We have here studied the role of Periheral Nerve Stimulation in degenerative lumbar spine pathologies with early neurological deficit, in which the patients were divided into test and control groups of 25 each.

Patients in the test group were treated with bed rest in the form of lumbar traction and medications while in the test group were treated with above as well as with PNS in addition to it.

Number of patients showing improvement i.e gaining back of power of MRC grade 5 was higher in test group as compared to control group which was also statistically significant with $\mathrm{p}$ value of $<0.047$.

Not only number of patients showing improvement was higher but also the duration of treatment required was less in test group with an average of approximately 8 days as compared to controls with an average duration of approximately 15 days, which was also statistically significant with $\mathrm{p}$ value of $<0.01$.

Thus, Peripheral nerve stimulation not only helps in improvement but also hastens the improvement in cases of degenerative lumbar spine pathologies, with early neurological deficit, thereby differing the decision of surgery in many of those cases which otherwise might have gone for operative management citing early neurological deficit as a cause.

Although ours was a kind of pilot study in demonstrating the role of Peripheral nerve stimulation in degenerative lumbar spine pathologies with early neurodeficit, it has still definitely showed its worth and can go on a long way in establishing it as a standard modality in conservative line of management in degenerative lumbar spine pathologies. Being a short duration study with a small sample size, it should be further revalidated with larger, randomized \& multi-SScentric study with a larger sample size for a longer duration for further proving its efficacy.

\section{References}

[1] Macnab's Backache: Textbook , $4^{\text {th }}$ ed.

[2] Campbells textbook of orthopaedics. $12^{\mathrm{t} h}$ edvol 2, pg1939

[3] Lower back pain and disorders of intervertebral discs Raymond J . Gardocki • ashley 1. Park. Chapter 42;

[4] Campbell's Textbook of Operative Orthopaedics.

[5] Electrotherapy explained, principles \& practice $3^{\mathrm{r} \mathrm{d}}$ edition (2000); low \& Ann Reed

[6] Cederwall E, Olsen MF, Hanner P, Fogdestam I (2006) Evaluation of a physiotherapeutic treatment intervention in "Bell's" facial palsy. Physiotherapy Theory Pract 22:43 - 52. [PubMed]

[7] Claytons electrotherapy, $9^{\mathrm{t} h}$ edition (2005) theory \& practice.. Angelaforster\& Nigel palastanga

[8] Farragher electrical stimulation for Bell's palsy. Clin Rehabil 1987;1:265 - 71

[9] Currier DP, Petrilli, ; Effect of graded electrical stimulation on blood flow to healthy muscle. Phys Ther,1986,Jun;66(6):937-43. 